\title{
Non-Destructive Evaluation Method for Soundness of Adhesive Portion Around Post-Installed Anchor Bolts in Concrete by Electromagnetic Pulse
}

\author{
[ Takayoshi Kimura, Shinya Uchida, Toshiro Kamada, Hirokazu Miyata ]
}

\begin{abstract}
This study focuses on the level of adhesive filling around a post-installed anchor bolts in concrete. The level of adhesive filling was evaluated in a concrete specimen simulating adhesiontype post-installed anchor bolts using an elastic wave method (electromagnetic pulse method). As a result, it is found that the level of adhesive filling around a post-installed anchor bolts can be evaluated accurately by inputting elastic waves in the bolt and using the elastic wave energy received on concrete surface near the bolt.
\end{abstract}

Keywords-Non-destructive testing, Post-installed anchor, Adhesive, Elastic wave method

\section{Introduction}

In the maintenance such as road construction, in the case of performing the inspection and investigation by method relying only to the sense of human sense, there is a possibility that variations in the results obtained. There are also issues such as it is difficult to manage the history and to quantitatively record the information obtained. From these, the introduction of inspection technology by non-destructive evaluation is desired for improved reliability and sophisticated inspection.

Especially, the technology development is urgently needed for properly maintain post-installed adhesive anchor bolt used for hang the heavy construction at the tunnel.

Against the above background, the authors, aiming at evaluating the level of adhesives filling around a post-installed anchor bolts in concrete, quantitatively and non-destructively did a study concerning the analysis-aided elastic wave method.

As a result, it is found that the level of adhesive filling around a post-installed anchor bolts may be evaluated accurately by inputting elastic waves in the direction of bolt axis and using the elastic wave energy received on concrete surface near the bolt.

Takayoshi Kimura/Master's Student

Ritsumeikan University

Japan

Sinya Uchida/Lecturer

Ritsumeikan University

Japan

Toshiro Kamada/Professor

Osaka University

Japan

Hirokazu Miyata/Researcher

West Nippon Expressway Company Limited

Japan
In this study, in order to verify the validity of the method proposed based on the analysis results, went a measurement using specimen with varying filling rate of the adhesive as a target based on electromagnetic pulse method.

\section{Electromagnetic Pulse Method}

The electromagnetic method is outlined in Figure 1[1]. In this method, the ferromagnetic material like a steel bar or anchor bolt in concrete are vibrated under a non-contact condition using the electromagnetic force produced as a magnetic field is generated instantaneously around an exciting coil (e.g., a magnetic steel sheet (iron core) wrapped with magnet wire), when electric current is applied to the exciting coil. The elastic waves caused by the vibration of the ferromagnetic material are caught by a vibration sensor installed on the surface of target member and waveform is analysed. Thus, defects on interface of ferromagnetic material and adhesion state can be evaluated by non-destructive manner.

\section{Overview of Experiment}

Figure 2 outlines the specimen. The concrete section measures $1000 \mathrm{~mm}$ in width, $1000 \mathrm{~mm}$ in length and $350 \mathrm{~mm}$ in thickness. A 24-mm-diameter hole was drilled in the concrete to a depth of $130 \mathrm{~mm}$. Then, a 240-mm-long anchor bolt (M16, SS400 equivalent material) was installed with a 110-mm-long part protruding from concrete surface. Adhesive (epoxy resin) was subsequently poured into the hole to fix the bolt. Adhesive filling patters are shown in Figure 3. Four levels of adhesive filling $(25,50,75$ and $100 \%)$ were used in the experiment. The level of adhesive filling is defined as the

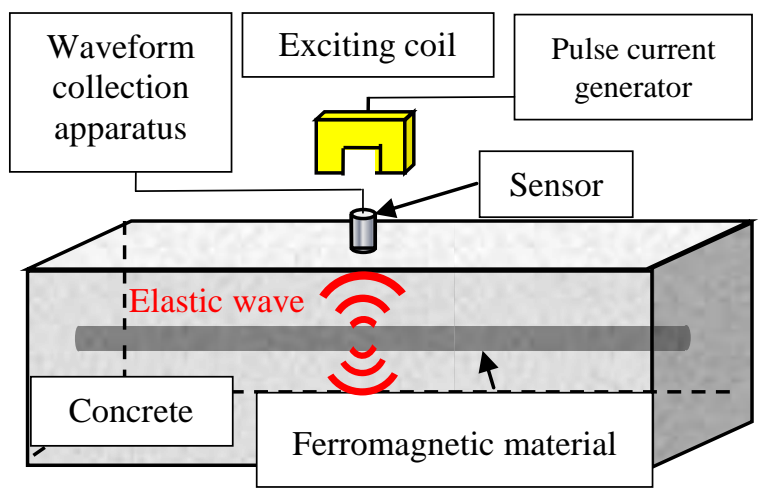

Figure 1. Outline of electromagnetic pulse method. 
ratio of the depth of hardened adhesive to the total depth of the

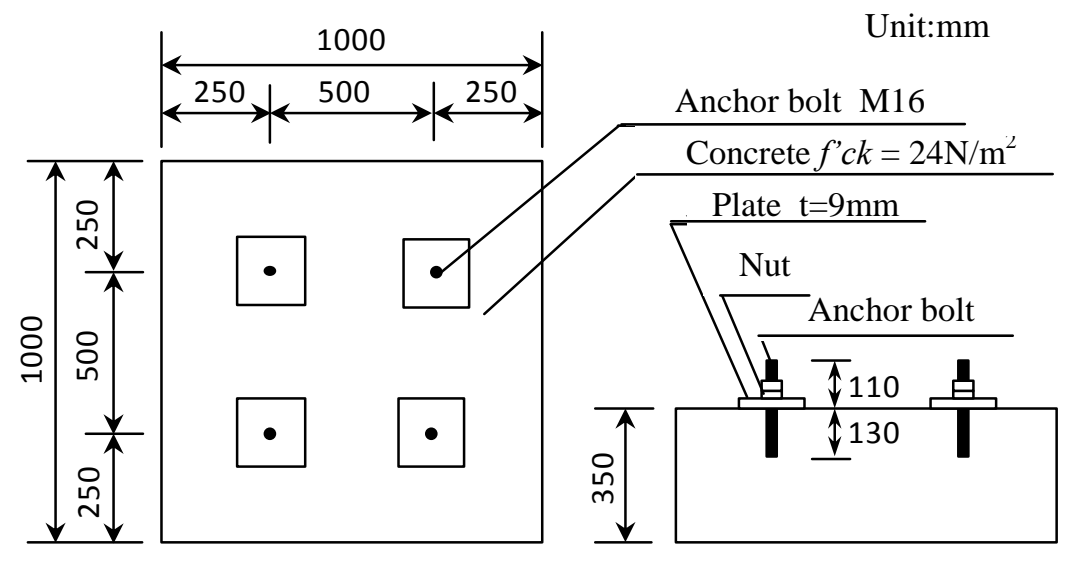

Figure 2. Outline of specimen.

hole. Specifically, at a level of adhesive filling of $25 \%$, adhesive was injected into the hole to a level $32.5 \mathrm{~mm}$ above the bottom of the hole. Four anchor bolts with varying adhesive filling levels were installed in one specimen (Figure 2). The design strength of concrete was set at $f_{c k}^{\prime}=24 \mathrm{~N} / \mathrm{mm}^{2}$. No ferromagnetic material (reinforcement) other than the anchor bolt was installed in the specimen. For each anchor bolt, a 100-mm-square, 9-mm-thick steel plate was fixed with two nuts.

Photograph 1 shows the view of measurement. The exciting coil was shaped like a cylinder with an outer diameter of 35 $\mathrm{mm}$ and an inner diameter of $31 \mathrm{~mm}$. An anchor bolt was made to penetrate the air core at the center of the coil, and the top ends of the coil and bolt were made to be in agreement with each other. A magnetic field was generated by instantaneously applying intensive electric current (approximately 3200A) around the ring-shaped exciting coil to cause the anchor bolt to vibrate. The elastic waves generated by the vibration of the bolt were received by a vibration sensor installed on concrete surface $100 \mathrm{~mm}$ away from the bolt center (Photograph 1). The signals received by the sensor were digitized at a sampling frequency of $2 \mathrm{MHz}$ and recorded by a waveform collection apparatus. Wave energy (sum of squares of amplitudes) was calculated for the waveforms recorded. The response sensitivity of the vibration sensor used is in the region between 15 and $100 \mathrm{kHz}$. The sensitivity characteristic is relatively flat in the region between 25 and 80 $\mathrm{kHz}$ in particular. Measurements were made ten times at each level of adhesive filling to grasp the fluctuation of evaluation parameters. In order to identify the basic vibration characteristics of bolts, experiments were conducted either in the case where neither nut nor plate was installed or in the case where both were installed and the bolt was fastened by 80 $\mathrm{N} \cdot \mathrm{m}$ using a torque wrench.

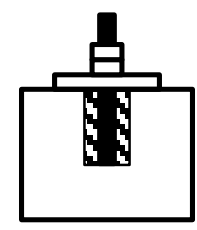

$100 \%$

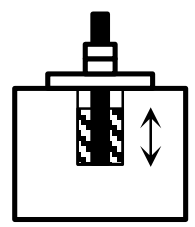

$75 \%$

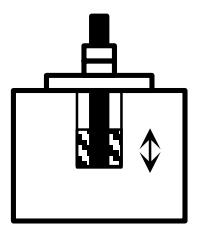

$50 \%$

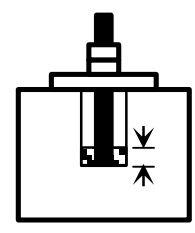

$25 \%$
Figure 3. Adhesive filling patterns.

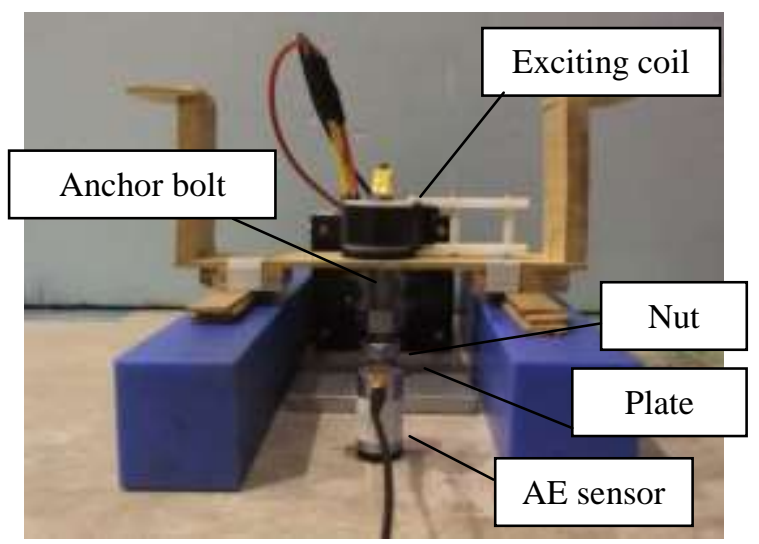

Photograph 1. View of measurement. 
Proc. of The Third Intl. Conf. On Advances in Civil, Structural and Mechanical Engineering - ACSM 2015

Copyright (C) Institute of Research Engineers and Doctors, USA .All rights reserved.

ISBN: 978-1-63248-083-5 doi: 10.15224/ 978-1-63248-083-5-64

\section{Experimental Results and}

\section{Discussions}

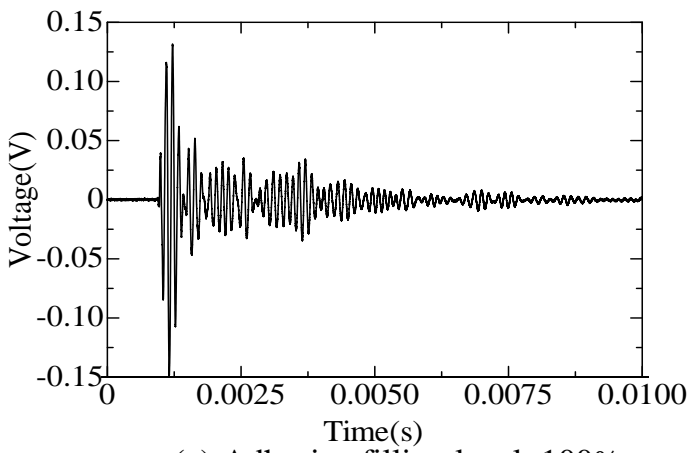

(a) Adhesive filling level: 100\%

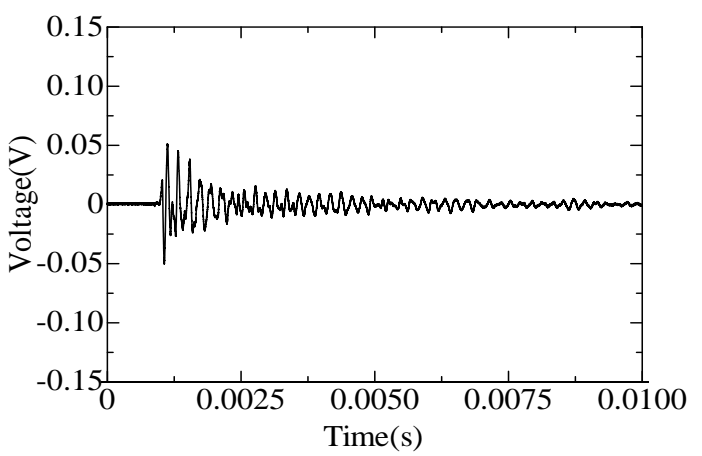

(c) Adhesive filling level: $50 \%$

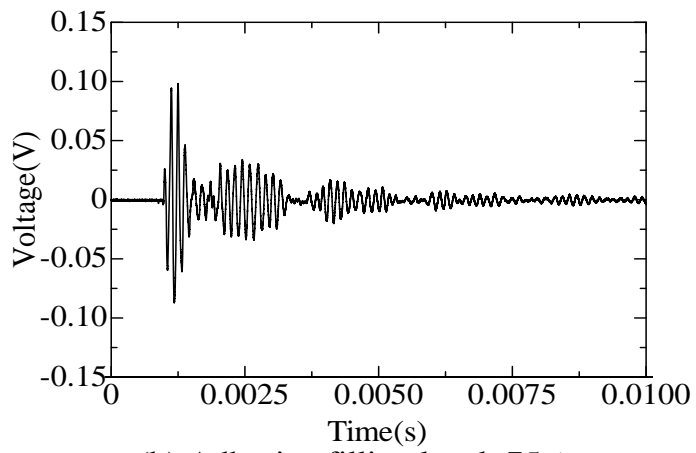

(b) Adhesive filling level: $75 \%$

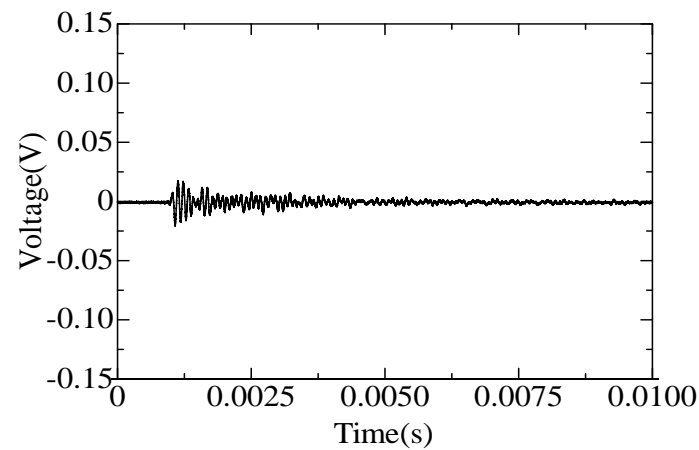

(d) Adhesive filling level: $25 \%$

Figure 4. Examples of received waveform.

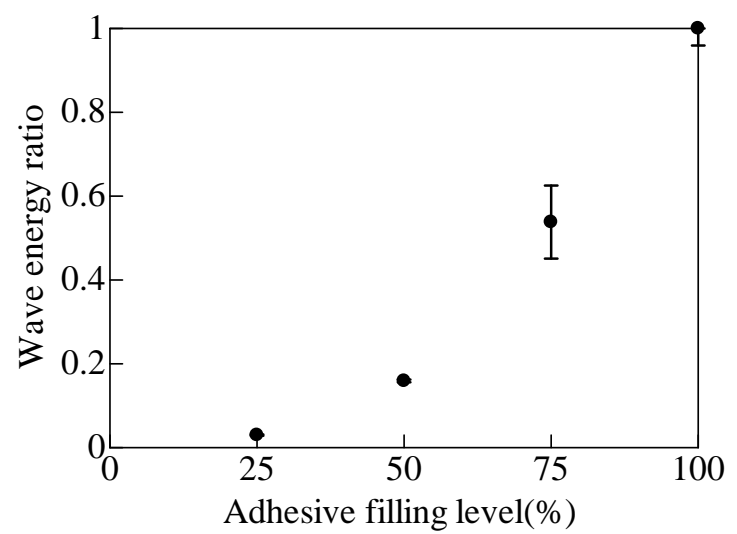

Figure 5. Relationship between wave energy ratio and adhesive filling level.

\section{A. Basic vibration characteristics of bolt}

Figure 4 shows waveforms received at varying adhesive filling levels. The figure confirms that the amplitude of waveform varies according to the level of adhesive filling. The maximum amplitude of waveform tended to decrease with the level of adhesive filling.
Figure 5 shows the relationship between wave energy ratio and adhesive filling level. The figure shows the ranges of mean, maximum and minimum measurements at each filling level. Wave energy tends to increase with filling level. This may be ascribable to the difference in attenuation while elastic waves propagated from the anchor bolt to concrete due to changes in the size of void. 


\section{B. Results obtains where the plate was installed and the bolt was fastened with nuts}

Figure 6 shows the relationship between wave energy ratio and adhesive filling level. It is evident that wave energy increased as the adhesive filling level increased. Wave energy ratio was, however, higher at a filling level of $75 \%$ than at $100 \%$. It was therefore found that recognizing the difference in adhesive filling level based on the wave energy was difficult at high filling levels. This is ascribable to easy propagation of elastic waves from the plate to concrete because of the increase in pressure on the plane where the plate was in contact with concrete due to the fastening by nuts (Figure 7). Based on the discussions above, it was considered important to make elastic waves of high amplitude to propagate through the

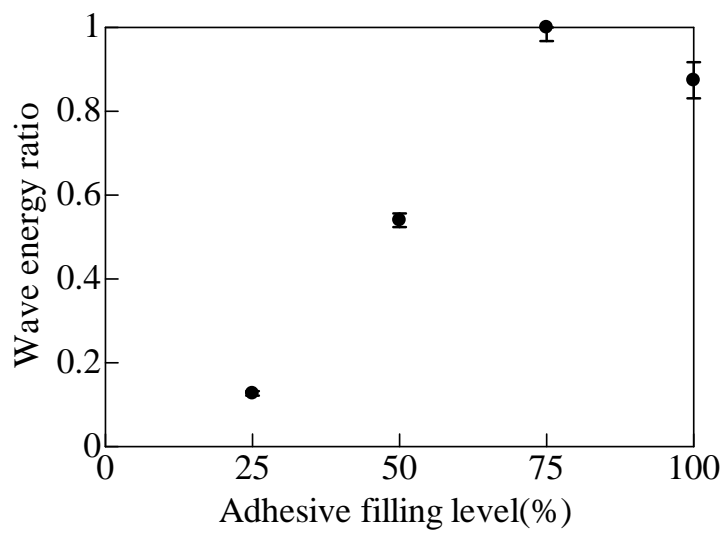

Figure 6. Relationship between wave energy ratio and adhesive filling level (fastened by nuts).

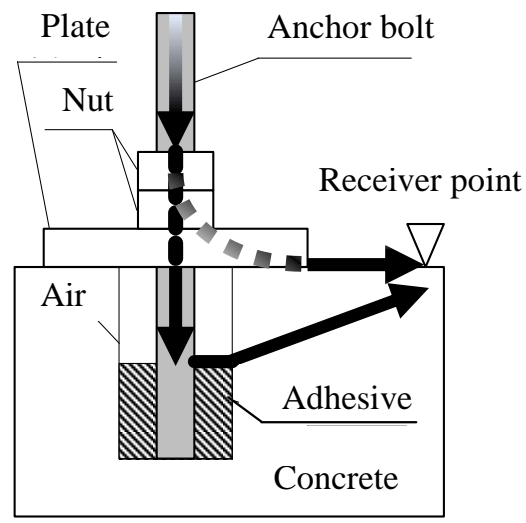

Figure 7. Image of elastic wave propagation when bolt was fastened by nuts. anchor bolt embedded in the concrete, for evaluating the level of adhesive filling. Then, measurements were made after strengthening the magnetic field. Specifically, a dynamic magnetic field was created using the exciting coil in the case where a static magnetic field was given by installing a neodymium magnet (permanent magnet) at the head of the anchor bolt to propagate_elastic waves through the bolt. Photograph 2 shows the outline of measurements. The measurement conditions are the same as in Section 4.1 except that a permanent magnet is used. Some of the waveforms obtained are shown in Figure 8. The figure shows that amplitudes are nearly ten times as large as for waveforms shown in Figure 4 regardless of the adhesive filling level. The wave energy ratios that were obtained using the measurement method are shown in Figure 9. It was revealed that waveform energy increased at a certain rate depending on the adhesive filling level.

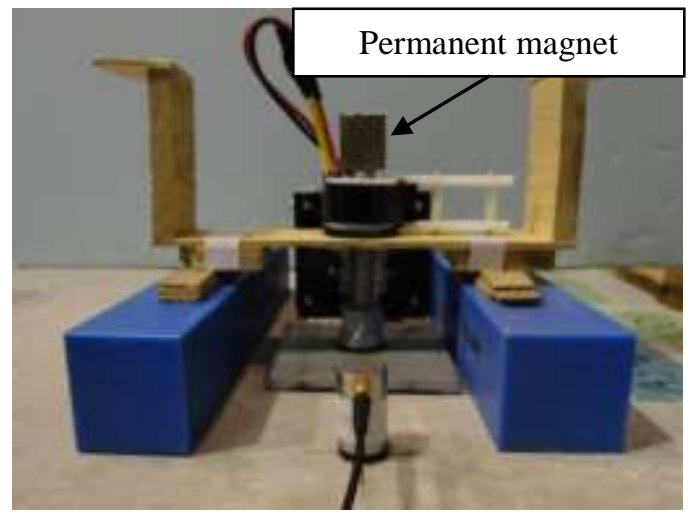

Photograph 2. View of measurement with permanent magnet.

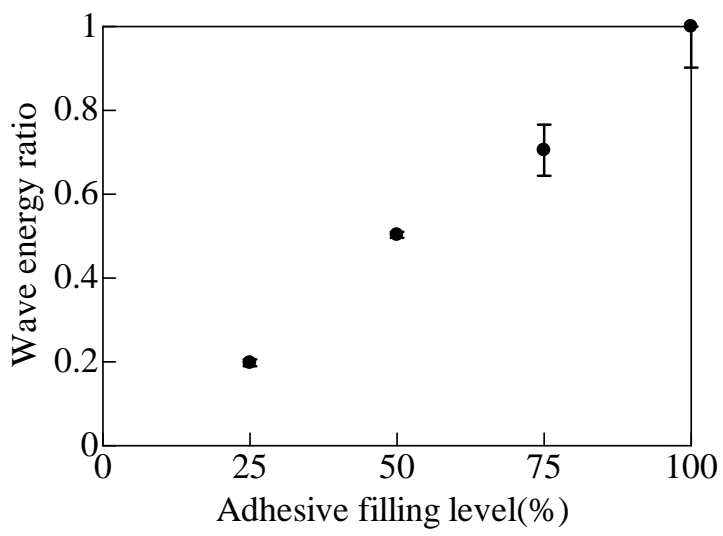

Figure 9. Relationship between wave energy ratio and adhesive filling level(with permanent magnet). 
Proc. of The Third Intl. Conf. On Advances in Civil, Structural and Mechanical Engineering - ACSM 2015

Copyright $(\odot$ Institute of Research Engineers and Doctors, USA .All rights reserved.

ISBN: 978-1-63248-083-5 doi: 10.15224/ 978-1-63248-083-5-64

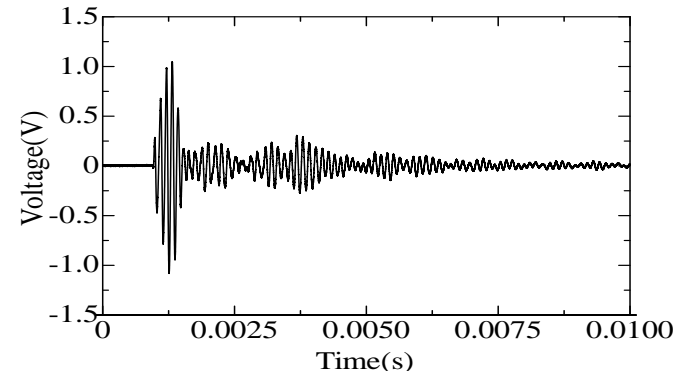

(a) Adhesive filling level: $100 \%$

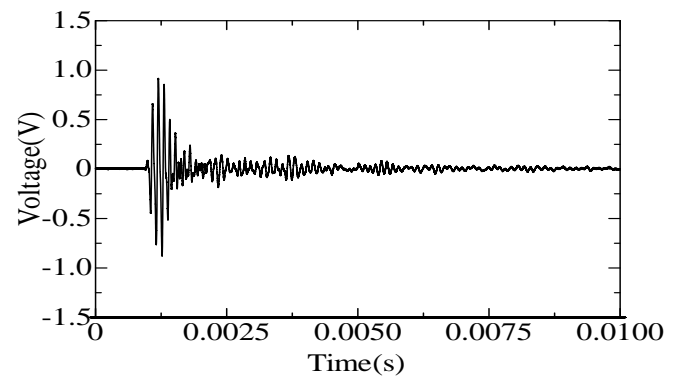

(c) Adhesive filling level: $50 \%$

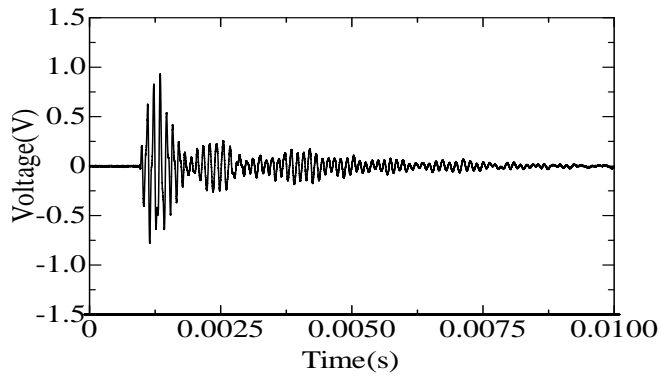

(b) Adhesive filling level: 75\%

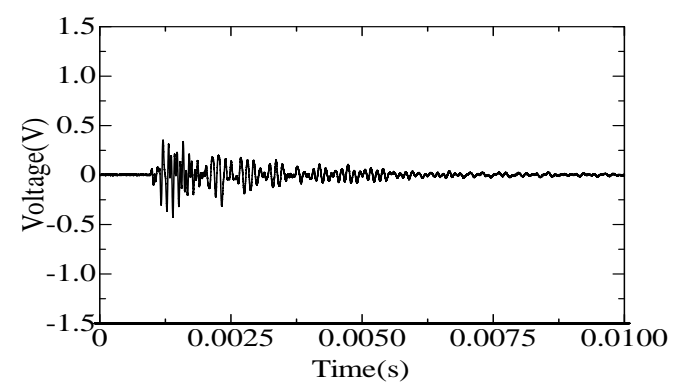

(d) Adhesive filling level: 25\%

Figure 8. Examples of received waveform (with permanent magnet).

\section{v. Conclusions}

The conclusions obtained in this study are described below. As a result of measurement made by a method determined based on the results of analysis;

(1) It was found that there was a correlation between the elastic wave energy received and adhesive filling level in the case where the plate was installed at the bolt and nuts loosened, which could be used to evaluate the level of adhesive filling.

(2) It was difficult to evaluate adhesive filling level in the case where the bolt was fastened with nuts.

(3) It was revealed that the difference in adhesive filling level around post-installed anchor bolts could be accurately identified based on the elastic wave energy by strengthening the magnetic field using a permanent magnet (static magnetic field).

\section{Acknowledgment}

The authors gratefully acknowledge the financial support of the Japan Society for the Promotion of Science KAKENHI Grant Numbers 25289132, 25820194.

\section{References}

[1] Munakata, K., Kamada, T., Uchida, S., Mae, H., \& Minezawa, H. (2009) Nondestructive evaluation of deterioration around rebar based on elastic waves generated by electromagnetic force. In: Proc. 7th Int. Symp. on Non Destructive Testing in Civil Engineering (pp. 107-112). Nantes, France.

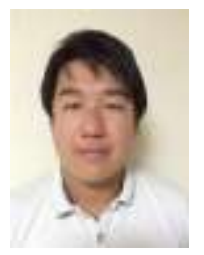

Takayoshi Kimura

Master's Student

Ritsumeikan University 